\title{
Performance Analysis of Quorum Based Power Saving Protocols for Ad hoc Networks
}

\author{
Ms.S.Kanimozhi, Mrs.M.Parameswari \\ (Communication systems,Dhanalakshmi Srinivasan Engineering College/Anna University,India) \\ (Communication systems,Dhanalakshmi Srinivasan Engineering College/Anna University,India)
}

\begin{abstract}
Energy is a heart of ad hoc networks. The lifetime of the network depends on the durability of the mobile node's battery power. Many energy conserving schemes are used to improve the energy efficiency and prolong the operational time of the mobile stations. Among these schemes Quorum based power saving protocol provides better energy conservation. In existing QPS the cycle length is identical to all the stations. The amount of conserved energy also low. To overcome this we propose a new scheme called Hyper quorum system (HQS). In this it uses an arbitrary cycle length for stations to maximize the energy efficiency and reduce the overall delay. Simulation results showed that the benefits of HQS over existing QPS.
\end{abstract}

Keywords: Ad hoc network, DCF, MAC, Power Saving Mode, Quorum systems

\section{Introduction}

Manet is formed by collection of mobile nodes without any pre planed infrastructure. Manet is self organized and dynamic in nature. It is mainly used in Military applications, Commercial uses and Personal area networks. Because of dynamic nature more energy is needed for ad hoc communication. One of the critical issues for Manet is power saving. A node is useless without power. The battery of the node is provide only limited energy so energy saving is an important one. Energy conservation can be achieved at different layers with different techniques. In physical layer when a station not transmitting means it goes to idle state. Even though it continuously listening the transmission. So idle listening consume more energy. To address this problem, power management policy is used. It is adopted at MAC layer, instead of idle listening it allows the station to sleep mode if there is no data transmission.

The IEEE 802.11 provide a solution for this problem. In IEEE 802.11 PSM include this mechanism which allows the station to sleep when there is no data transmission. It divides the time into beacon interval. At the beginning of each beacon interval all the station must wake, if there is no transmission means it goes to sleep mode. IEEE 802.11 has two mechanism to access the channel. 1) PCF 2) DCF. Where PCF is contention free and centralized control.

DCF is based on CSMA/CA and fully distributed in nature. In this paper we consider a PSM in DCF mechanism. The main drawback of PSM is at every beacon interval it wake up continuously and consume more energy.

To overcome this we go for Quorum systems. In all existing QPS protocols, the cycle length is either equal for all stations or is limited to certain numbers . These boundaries on cycle length severely limit the practical use of QPS protocols as each individual station may desire to select a cycle length that is best suited for its own need .In this paper, we propose the idea of hyper quorum system (HQS) - a generalization of QPS that allows for arbitrary cycle lengths. By using the HQS we yields $41 \%$ improvement in energy efficiency and $90 \%$ decrement in delay.

1) IEEE 802.11 power saving mode:

\section{Preliminaries}

As mentioned earlier, time is divided into successive beacon intervals. At the beginning of each beacon interval, hosts will compete to send a beacon frame, which is used for clock synchronization. Each host generates a arbitrary delay and waits for the duration of that delay. A host will cancel the random delay timer before it has expired, if a beacon is received from another host. Otherwise, it will send a beacon when the random delay timer has expired. A host operating in IEEE 802.11 PSM can either be in the awake mode or the sleep mode. The host is completely powered in the awake mode and, thus, can transmit or receive at any time, whereas when a host is in the sleep mode, it cannot transmit/receive packets. A host must be awake during the ATIM window, which is situated at the beginning of every beacon interval, to ensure if it has any pending packets. If so, the host must remain awake for the remainder of the time. A host with packets pending for another host must first make an announcement during the ATIM window. This announcement is accomplished by sending an ATIM frame. Each host monitors these announcements 
to choose whether it should stay awake for the rest of the beacon interval. Upon receiving an ATIM frame, the receiving host must reply with an ATIM-ACK frame. A host not receive an ATIM frame it can switch to sleep mode at the end of the ATIM window. Actual data transfer is accomplished after the ATIM window. Fig. 1 illustrates this IEEE 802.11 PSM. In the first beacon interval, host A has effectively broadcast a beacon. After the ATIM window, all hosts switched to sleep mode, since no host had to transmit packets. In the second beacon interval, after host $\mathrm{C}$ had broadcast a beacon, host B sent an ATIM frame to host C during the ATIM window and received an ATIM-ACK frame from host $\mathrm{C}$ as the acknowledgement. Hosts $\mathrm{B}$ and $\mathrm{C}$ both remained awake for the entire beacon interval. After the ATIM window, host B started its data transmission to host C.

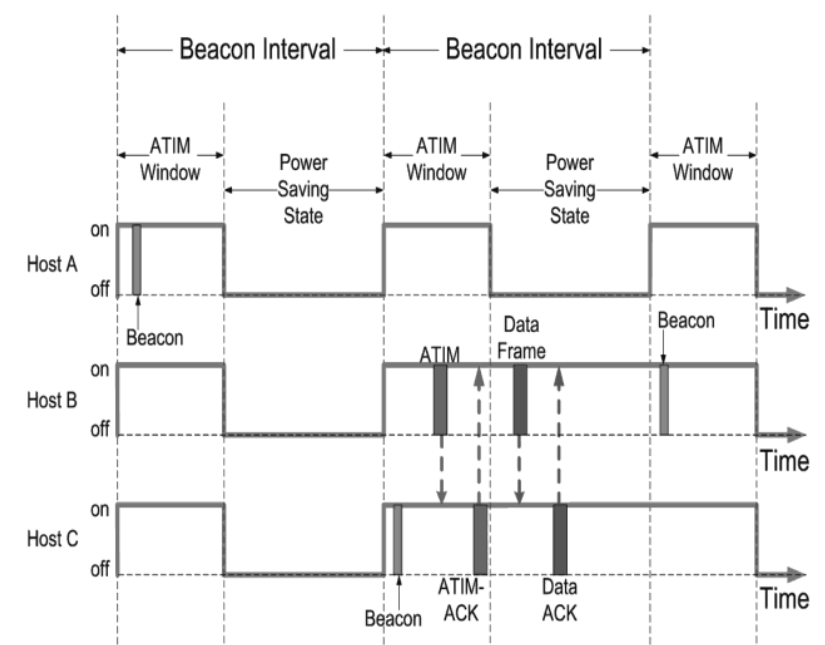

Fig.1. Power saving mechanism in IEEE 802.11.

\section{2) Problem statement:}

A host in the IEEE 802.11 PSM must wake up at every beacon interval. So it is stay awake for the duration of the ATIM window. After that only it goes to sleep mode if it does not have any transmission. Therefore it have continuous sleep to awake and awake to sleep switches, it make an excess amount of unnecessary energy consumption. To solve this problem we go for quorum systems.

\section{3) Quorum concepts:}

A quorum is a collection of sets that intersects with each other at least once within a certain period. The quorum concepts widely used in mutual exclusion, fault tolerance and read / write applications. There are many kinds of quorum, such as grid, cyclic and etc, here we use the quorums to identify the beacon intervals, during which a host must wake up. Without loss of generality, we used a grid based quorum to implement our protocols. In a grid quorum, one row and one column is picked in an $\mathrm{n} \times \mathrm{n}$ grid. Fig 2 illustrates this concept. Here host A picked row Ra and column Ca as its quorum, while host $\mathrm{B}$ picked row $\mathrm{Rb}$ and column $\mathrm{Cb}$. There are two intersections between hosts $\mathrm{A}$ and $\mathrm{B}$.

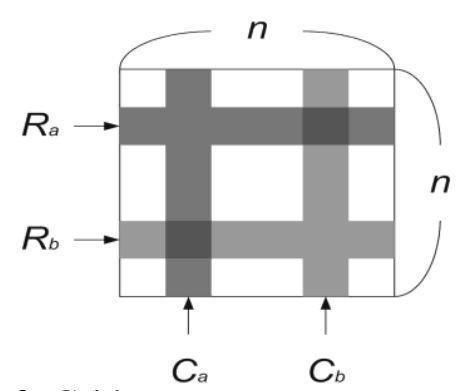

Fig 2. Grid quorum system example.

\section{Protocols Description}

1) Adaptive Quorum based Energy Conserving Protocol(AQEC)

In QEC (Quorum based Energy Conserving) protocol the grid size is $\mathrm{n} \times \mathrm{n}$. We cannot change the grid size. But the selection of grid size is valuable one. A large grid size provides more power saving with larger 
delays. On the other hand, the amount of conserved energy is reduced with smaller grid size. For example when the grid size is reduced to $1 \mathrm{x} 1$ means the QEC is similar to IEEE 802.11 PSM.

In order to achieve better performance it is necessary to dynamically change the grid size for individual stations. It is achieved by employing the AQEC. The idea behind AQEC is to increase the grid size to prolong the station's sleep duration in light traffic and decrease the grid size to wake up the station for heavy traffic. In AQEC the cycle length values are 4,9,16 which is selectable for a station. In this we cannot increase the cycle length. The simple example of $\mathrm{AQEC}$ is given below.

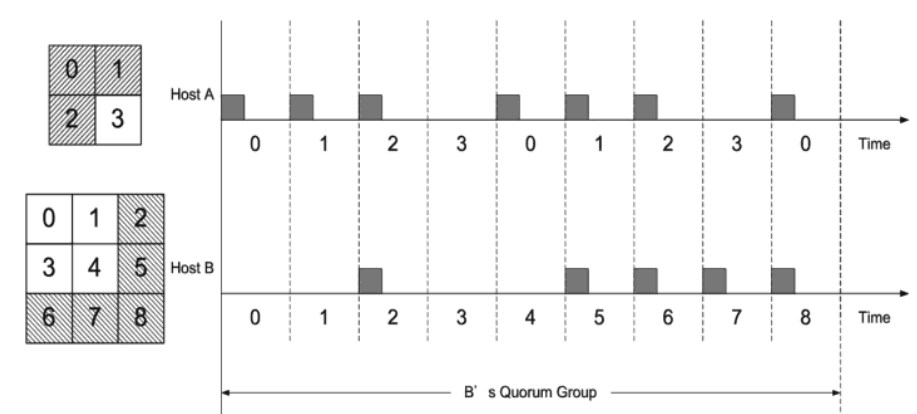

Fig. 3. Intersections of Host A and Host B.

Here we consider two hosts with different grid size will intersect with each other. Host A has $2 \times 2$ grid and its quorum intervals are $0,1,2$. Host $\mathrm{B}$ has $3 \times 3$ grid size and its quorum intervals are 2,5,6,7,8. Host $\mathrm{A}$ wakes up more frequently than Host B but they intersects at host B quorum group.

\section{2) Hyper Quorum Systems(HQS)}

HQS is a generalization of traditional quorum systems, where the stations may obtain cycle patterns of arbitrary lengths that best suit their requirements. The advantages of hyper quorum system are 1) HQS allows more energy saving for the stations even though the critical battery power. 2) HQS provides a controllable delay. We use two algorithms to build HQS , they are

1) semi adaptive grid 2) difference set. These two are fully adaptive in adhoc environment.

1) Semi adaptive grid:

We consider an extended grid quorum $\mathrm{G}$ over the modulo-n it is given by $\mathrm{Gi}=\{0,1 \ldots \ldots . . \mathrm{n}-1\}$. Here we consider the square grid.

\section{2) Difference set:}

We consider a quorum $\mathrm{D}$ over the modulo-n it is given by

$\mathrm{Di}=\{0,1, \ldots . \mathrm{n}-1$.$\} . This set define the interspacing between the elements.$

In the hyper quorum systems we can select the random value of cycle length. Here the cycle length of HQS was showed. Fundamentally the HQPS protocol defines two different cycle patterns. In synchronous cycle the stations awake during the ATIM window. The diagram for synchronous cycle patterns are shown in Fig. 4(a) and 4(b). Alternatively the station choose the asynchronous cycle pattern as depicted in Fig 4(c) and 4(d).

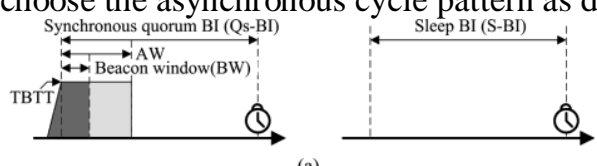

(a)
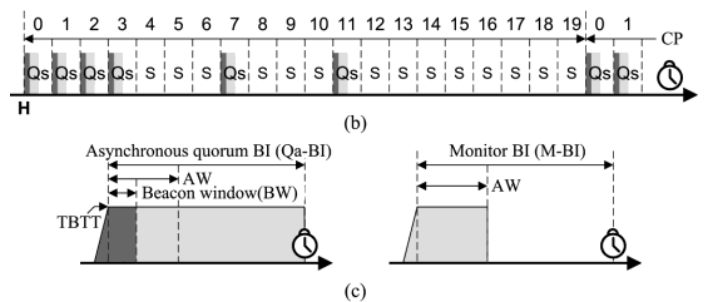

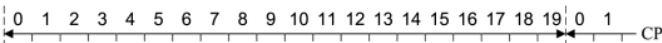

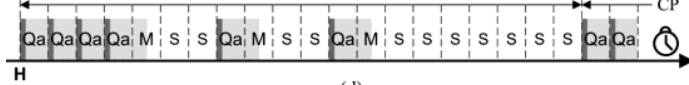

Fig. 4. HQPS protocol 


\section{Performance Analysis}

In this section we evaluate the performance of $\mathrm{HQS}$ with $\mathrm{AQEC}$ protocol. We implement our simulation based on the ns-2 simulator with CMU wireless extension. The simulation is conducted in $400 \mathrm{~m} \mathrm{x}$ $400 \mathrm{~m}$ static network with 50 randomly distributed stations. Each station has half - duplex wireless channel of rate $2 \mathrm{Mbps}$ and the transmission range of $100 \mathrm{~m}$. we set the duration of beacon interval as $100 \mathrm{~ms}$ and ATIM window as $25 \mathrm{~ms}$. The packet size is $512 \mathrm{kbps}$. The power consumption for transmit mode is $1650 \mathrm{~mW}$, for receive mode $1400 \mathrm{~mW}$, for idle mode $1150 \mathrm{~mW}$ and for sleep mode $45 \mathrm{~mW}$. Here we take the HQPS in synchronous environments.

\section{1) Alive node calculation}

In this we evaluate the number of alive nodes in each protocols. Compare with AQEC the HQPS have minimum number of alive nodes are high. If the number of alive nodes are high means the lifetime of the network also high.

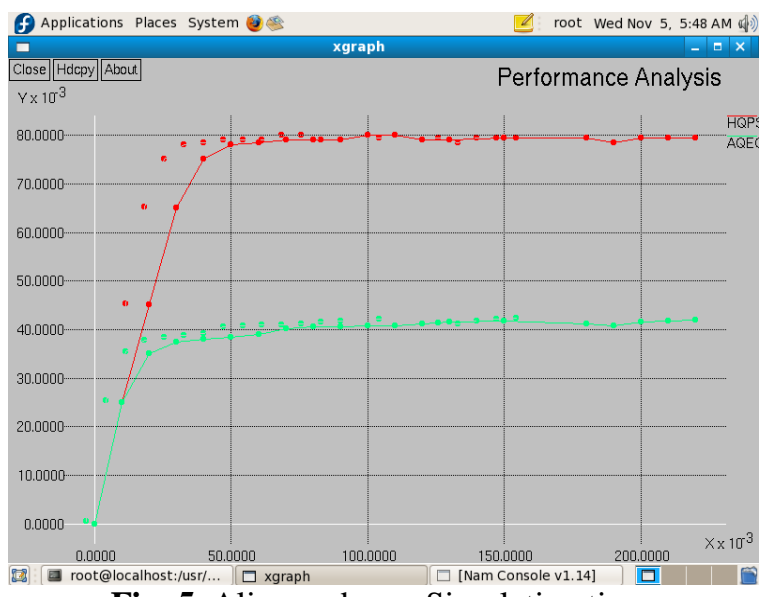

Fig. 5. Alive nodes vs Simulation time

\section{2) Link Discovery}

In this section we study the link discovery time. In practice the AQEC and HQPS work quite well. The AAPM provide long link discovery than AQEC and HQPS.

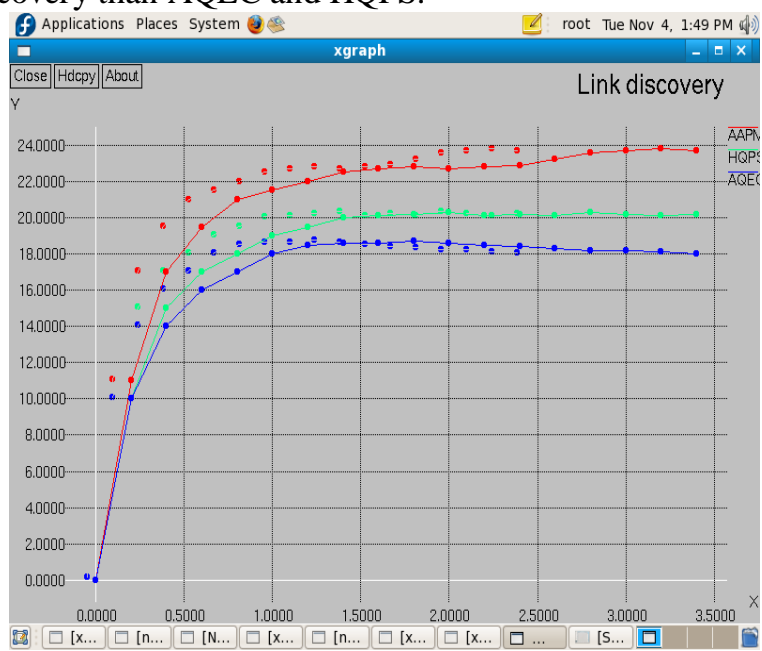

Fig.6.Link discovery ratio vs Simulation time

\section{3)Delay drop calcuation}

In this section we consider the average delay encountered during the packet transmission. 


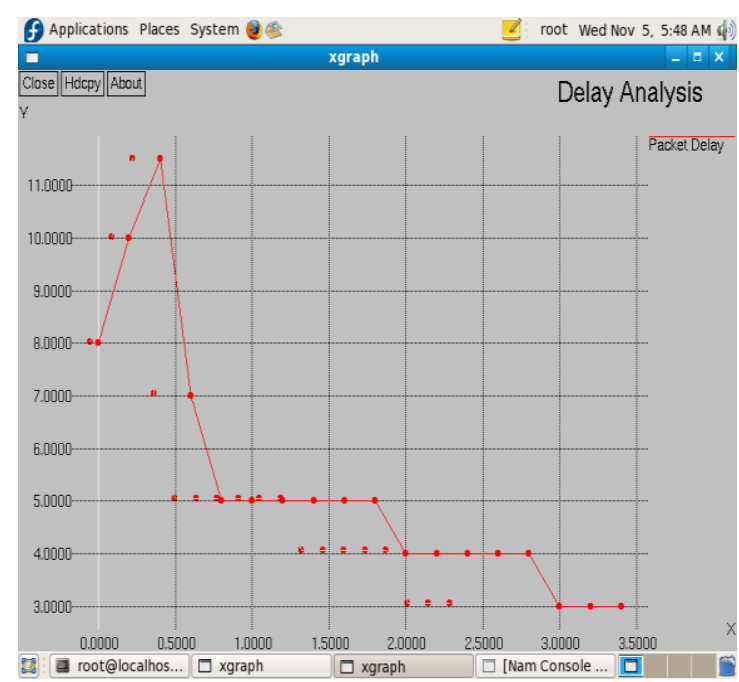

Fig.7. Delay drop ratio vs Traffic load

Under the light traffic the delay is high and for heavy traffic the stations are in awake state. So the delay is reduced by the use of HQPS protocols.

\section{4)Energy conservation}

In this section we evaluate the energy sufficiency of AQEC and HQPS protocols for different traffic loads. As compared with AQEC, HQPS provides more energy conservation at heavy traffic loads. By using HQPS we can conserve the energy up to $31 \%$ to $41 \%$. Initial energy for each node is $100 \mathrm{~J}$ at every $30 \mathrm{~s}$ the calculation is takes place.

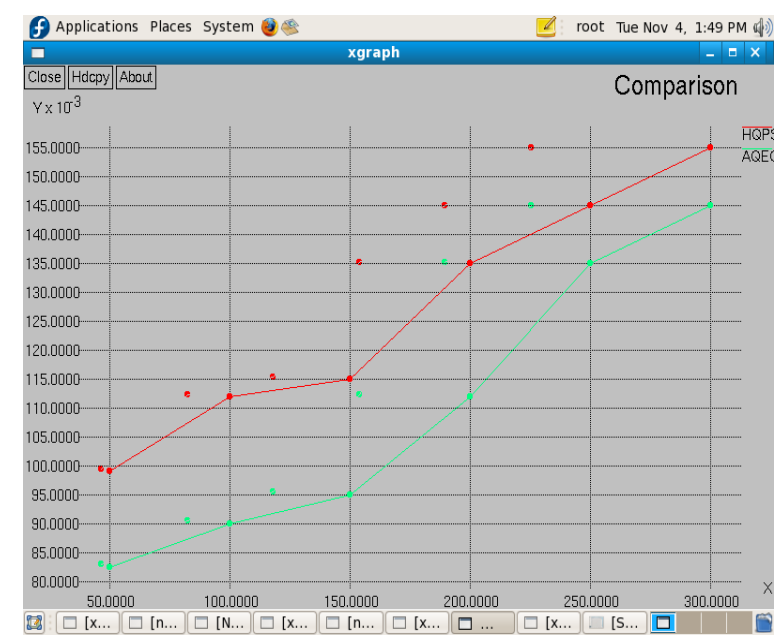

Fig. 8.Energy consumption vs Traffic load

\section{Conclusion}

Energy conservation is a critical issue in wireless network. In this paper we proposed HQS and it is fully adaptive it can select random cycle length to conserve more energy. Experimental results showed that our protocol gives significantly stable performance for various traffic loads. In particularly gives up to $41 \%$ of energy efficiency in heavy traffic and $90 \%$ delay drop in light traffic conditions. This concept is implemented only at MAC layer. In future work, we will try to implement this in cross layer technique. But still it is very big challenge in ad hoc networks.

[1]. The network simulator(ns-2)[online].

\section{References}

[2]. G.Bianchi, "Performance analysis of theIEEE 802.11 distributed co-ordination fuction", IEEE J. sel. Areas Commun., vol. 18, no. 3, pp. 535-547, Mar. 2000.

[3]. C. chao, J.Sheu, and I. Chou, "An adaptive quorum based energy conserving protocol for IEEE 802.11 ad hoc networks", IEEE Trans. Mobile Comput. Vol. 5, no. 5, pp. 560-570, May 2006.

[4]. I.L.S. Committee, "Wireless LAN medium access control and physical layer specifications", 1999.

[5]. E. Jung and N. Vaidya, “An energy efficient MAC protocol for wireless LANs', in Proc. IEEE INFOCOM,2002,pp. $1756-1764$. 
[6]. W.Luk and T.Wong,"Two new quorum based algorithms for mutual exclusion “, in Proc. ICDCS, 1997, pp. 100-106.

[7]. Y. Tseng, C. Hus and T. Heish, "Power saving protocols for IEEE 802.11 based multihop adhoc networks", in Proc.IEEE INFOCOM, 2002, pp. 200-209.

[8]. R.Zheng and R. Kravets, "On-demand power management for ad hoc networks", in Proc. IEEE INFOCOM, 2003,pp. 481-491.

[9]. Z. Chou, "Optimal adaptive power management protocols for asynchronous wireless ad hoc networks", in Proc. IEEE WCNC, 2007,pp. 61-65.

[10]. C. Perkins and E.Royer,"Ad hoc on demand distance vector routing”, in Proc. IEEE WMCSA, 1999, pp. 90-100. 